Opinion in Cell Biology

Manuscript Number: COCEBI-D-16-00002R1

Title: Signalling at Membrane Contact Sites: two membranes come together to handle second messengers

Article Type: SI:39: Cell Regulation (2016)

Corresponding Author: Dr. Tim Levine,

Corresponding Author's Institution: UCL Inst of Ophth

First Author: Tim Levine

Order of Authors: Tim Levine; Sandip Patel, PhD

Abstract: It is now clear that many intracellular signals result from multiple membrane-bound compartments acting in concert. Membrane contact sites, regions of close apposition between organelles, have emerged as major points of convergence during signaling, as these are places where material is exchanged. The material exchanged can be either waterinsoluble molecules such as membrane lipids that are passed directly between membranes, or ions such as Ca2+. Here we highlight new insights into the role of contacts in regulating several aspects of signaling by second messengers, including lipid traffic that underpins generation of IP3 during intense PLC stimulation, NAADP generated Ca2+ signals, storeoperated Ca2+ entry, and cyclic AMP signaling. 


\title{
Signalling at Membrane Contact Sites: two membranes come together to handle second messengers
}

Tim P Levine ${ }^{1 *}$ and Sandip Patel ${ }^{2 *}$

${ }^{1}$ UCL Institute of Ophthalmology, London EC1V 9EL, UK

${ }^{2}$ Department of Cell and Developmental Biology, University College London, London WC1E 6BT, UK

* to whom correspondence should be addressed:

tim.levine@ucl.ac.uk; patel.s@ucl.ac.uk

\begin{abstract}
$\underline{\text { Abstract }}$
It is now clear that many intracellular signals result from multiple membrane-bound compartments acting in concert. Membrane contact sites, regions of close apposition between organelles, have emerged as major points of convergence during signaling, as these are places where material is exchanged. The material exchanged can be either water-insoluble molecules such as membrane lipids that are passed directly between membranes, or ions such as $\mathrm{Ca}^{2+}$. Here we highlight new insights into the role of contacts in regulating several aspects of signaling by second messengers, including lipid traffic that underpins generation of $\mathrm{IP}_{3}$ during intense PLC stimulation, NAADP generated $\mathrm{Ca}^{2+}$ signals, store-operated $\mathrm{Ca}^{2+}$ entry, and cyclic AMP signaling.
\end{abstract}

\author{
Abbreviations \\ A-kinase anchoring protein - AKAP \\ diacylglycerol - DAG \\ endoplasmic reticulum - ER \\ extended synaptotagmin - E-Syt \\ nucleus-vacuole junction - NVJ \\ phosphatidic acid - PA \\ phosphatidylcholine - PC \\ phosphatidylinositol - PI \\ phospholipase C - PLC \\ PI phosphate - PIP \\ store-operated Ca2+ entry - SOCE
}




\section{Introduction}

The events of most signaling pathways are classically modelled as taking place in one subcellular compartment, for example the plasma membrane. However, even classical signaling pathways involve components of other organelles such as the endoplasmic reticulum (ER). This necessitates interorganellar or intermembrane cross-talk. While this could theoretically take place at a distance, it is now clear that coordination is facilitated by membrane contact sites, which we define as any place inside cells where organelles come close to each other (see Box 1) [1,2].

The more prominent membrane contact sites, such as those between the ER and mitochondria or between the ER and plasma membrane, have been observed since the earliest ultrastructural studies of intracellular architecture [3]. They have long been posited to be locations of important cellular functions [4,5], and they were eventually found to harbor specific proteins, such as $\mathrm{Ca}^{2+}$ channels and lipid biosynthetic enzymes. The field of contact sites has blossomed in the last decade, with many more contact site components being identified, although specific tethers that structure membrane contact sites remain mostly unknown $[1,2,6]$, with notable exceptions [7].

The presence of a membrane contact site has two major effects: (i) Enhancing speed of communication. A 30-fold reduction of distance (from 1 micron to $30 \mathrm{~nm}$ ) produces a 1000 -fold reduced diffusion time (from $200 \mathrm{~ms}$ to $200 \mu \mathrm{s}$ ); therefore, membrane contact sites are ideal places where signalling pathways can pass information and material between organelles. (ii) Creating biochemically distinct subdomains in each organelle where a unique set of functions co-exist; controlling entry/exit into these subdomains could be a way to regulate signalling. Here, we highlight a selection of the recent developments that link membrane contact sites to signalling by different second messengers.

\section{1. $\mathrm{Ca}^{2+}$ signaling at the plasma membrane and the phosphatidylinositol (PI) cycle.}

$\mathrm{Ca}^{2+}$ is a ubiquitous signalling ion that is well established in regulating cell function. Its versatility is underpinned by spatiotemporal complexity of the $\mathrm{Ca}^{2+}$ signal; in essence cells can sense (decode) where and when $\mathrm{Ca}^{2+}$ signals occur [8]. $\mathrm{Ca}^{2+}$ signal genesis can be thought of as a chain of events which start and end at the plasma membrane but involving $\mathrm{Ca}^{2+}$ fluxes from storage organelles, including the ER, acidic organelles, and mitochondria. At several steps, membrane contact sites between different $\mathrm{Ca}^{2+}$ sources are likely critical for communication. $\mathrm{Ca}^{2+}$ flux across the ER-mitochondria interface has been extensively studied [9]; here, we focus on a new aspect of $\mathrm{Ca}^{2+}$ signaling at ER-plasma membrane contacts.

A classical mechanism for generation of $\mathrm{Ca}^{2+}$ signals at the plasma membrane is through receptormediated activation of phospholipase C (PLC), which hydrolyzes $\mathrm{PI}(4,5) \mathrm{P}_{2}$ to $\mathrm{IP}_{3}$ and diacylglycerol (DAG), leading to release of $\mathrm{Ca}^{2+}$ from $\mathrm{ER} \mathrm{Ca}^{2+}$ stores by $\mathrm{IP}_{3}$ receptors. One long-suggested role for contact sites is the resupply of PI to the plasma membrane during intense PLC signaling [10], which occurs during prolonged stimulation with hormones and neurotransmitters. Under these conditions not only is all the PI $(4,5) \mathrm{P}_{2}$ hydrolyzed, but there is not enough PI in the plasma membrane to resynthesize the amount of $\mathrm{PI}(4,5) \mathrm{P}_{2}$ that is consumed [11]. Therefore, the plasma membrane needs PI to be resupplied. This occurs by recycling the lipid moiety of PI $(4,5) \mathrm{P}_{2}$. PLC makes DAG and this undergoes five modifications to be returned to PI(4,5) $\mathrm{P}_{2}$ (Figure 1). But the second and third of these modifications are carried out by ER membrane enzymes [12,13], the active sites of which are located in short ( $\leq 20 \mathrm{aa}$ ) loops on their cytosolic faces. Therefore, plasma membrane lipids $\geq 15 \mathrm{~nm}$ away [14•] cannot be substrates. This led to the proposal towards the end of the last millennium that the precursor lipid for these enzymes (phosphatidic acid, PA) and their product (phosphatidylinositol, PI) exchange at plasma membrane-ER contact sites [10]. Vindicating that prediction, it has now been shown that a single protein transfers both PA and PI in a countercurrent (Figure 1).

Massive and sustained $\mathrm{PI}(4,5) \mathrm{P}_{2}$ hydrolysis accompanies phototransduction in flies, but not in vertebrates. The retinal degeneration-type B (RdgB) protein is localised to contacts between the ER and plasma membrane in fly photoreceptors. It was known to transfer both PI and phosphatidylcholine (PC) like all other lipid transfer proteins in the same family, so it could carry out half of the necessary traffic in $\mathrm{PI}(4,5) \mathrm{P}_{2}$ resynthesis [15]. Nir2, a human homolog of RdgB, was 
elegantly shown to have a similar role during intense $\mathrm{Ca}^{2+}$ signaling [16] . However, no means for traffic of PA, the other half of the reaction, was known. Things started to change when RdgB was found to have a weak ability to solubilise PA [17]. Now the PI transfer domains in both RdgB and Nir2, have been shown to exchange PA for PI at plasma membrane-PM contacts during PLC activation (Figure 1) $[18 \bullet \bullet, 19 \bullet \bullet]$. The C-terminal domains in RdgB/Nir2 bind the PA headgroup, enhancing contact site targeting in response to PIP hydrolysis [20]. In addition Nir3, a close homolog of Nir2, has a higher affinity for the PA headgroup and slower transfer of PA, so that it can exchange lipid after low level PIP hydrolysis [21•]. Thus, there is a series of PA/PI exchange proteins fine-tuned to be recruited and act during different intensities of PLC activation to resupply lipid for $\mathrm{IP}_{3}$ generation.

The mechanistic details of lipid transfer during PA/PI exchange has not yet been confirmed. It has been proposed that lipid modifying enzymes use lipid transfer proteins as co-factors to present (and activate) lipid [22]. This might apply for PI to PI(4)P conversion, but PI(4)P cannot bind RdgB/Nir, so this lipid must be present in the plasma membrane (Figure 1). The possibility of lipid transfer proteins acting as enzyme cofactors could be tested, for example by looking for direct binding of lipid transfer protein to enzyme. Overall, the important take-home message from the new work is that subtle variation in lipid specificity [17] and affinity [21•] allows closely related lipid transfer proteins to perform specific functions.



In addition to $\mathrm{IP}_{3}$, the second messenger NAADP plays an important role in generating highly localised $\mathrm{Ca}^{2+}$ signals [23]. This messenger is unusual as it activates $\mathrm{Ca}^{2+}$ release from acidic organelles such as lysosomes. Its likely targets are two-pore channels (TPCs), ancient members of the voltage-gated ion channel super family [24]. Importantly, the $\mathrm{Ca}^{2+}$ signal from these stores is amplified and propagated by $\mathrm{Ca}^{2+}$ release channels on the ER such as the $\mathrm{IP}_{3}$ receptor, even when NAADP is bypassed by releasing lysosomal $\mathrm{Ca}^{2+}$ directly through osmotic stress [25]. Despite much evidence for interorganellar communication, we know little concerning how signals are transmitted across the acidic organelle-ER interface. Indirect evidence supports an intimate connection. For example, functional coupling is lost in is several unrelated cell types when cells are broken open; presumably the geometry of $\mathrm{Ca}^{2+}$ stores is disrupted. TPC localization is also critical, as TPCs do not trigger $\mathrm{ER} \mathrm{Ca}^{2+}$ release when rerouted to the plasma membrane [26]. Cross-talk at membrane contact sites has been an attractive explanation for "tight" functional coupling [27], but little is known about these sites.

What is the hard evidence in support of membrane contacts between lysosomes and the ER? They have only recently been studied at the ultrastructural level $[25,28,29]$. However, unlike their endosome-ER counterparts slightly earlier in the endocytic pathway, their molecular composition remains undefined. Thus, a formal demonstration that lysosome-ER contacts support lysosome-ER $\mathrm{Ca}^{2+}$ communication is at present lacking. Drawing on the morphological similarity between the nucleus-vacuole junction (NVJ) in yeast and lysosome-ER junctions in animal cells, one might anticipate similarities in composition [27]. The founding NVJ tethers (vacuolar Vac8p and nuclear Nvj1p) are not conserved in animals [7]. However, other NVJ components are conserved widely: Nvj2p [30], Lam6p/Ltc1p [31] and Mdm1p and Nvj3p [32••]. Although it has been proposed that Lam6p is also a component of mitochondrial-vacuolar contacts [33], as we have argued elsewhere Lam6p is an ER membrane protein; therefore Lam6p near mitochondrial-vacuolar contacts must be in an ER tubule very close to the vacuole [31,33]. A similar peri-vacuolar (but non-NVJ) distribution has been found for Mdm1p and Nvj3p [32••]. This raises the possibility that some of the Lam6p/Mdm1p/Nvj3p-positive structures correspond to direct contacts between the yeast vacuole and ER tubules. Such contacts have never been described before in yeast, and so we suggest the name Vacuolar $\underline{\mathbf{n}}$ on-NVJ $\underline{\text { cytoplasmic }} \underline{\mathbf{E}}$ (VancE) contacts. These may well be orthologous to lysosome-ER membrane contact sites in animal cells, which would indicate ways in which the yeast model system can be used to study acidic $\mathrm{Ca}^{2+}$ stores. The finding that SNX14, a human homologue of Mdm1p and Nvj3p in yeast, is required for normal neuronal cell function [34] indicates that studies characterizing its roles in lysosomal-ER contact formation and function in animal cells are urgently required. 


\section{Membrane contact sites and the regulation of store-operated $\mathrm{Ca}^{2+}$ entry.}

Store-operated (or capacitative) $\mathrm{Ca}^{2+}$ entry (SOCE) is a well established phenomenon linking depletion of $\mathrm{ER} \mathrm{Ca}^{2+}$ stores to entry of $\mathrm{Ca}^{2+}$ across the PM. Its debut in the membrane contact site world rested on molecular identification of the ER $\mathrm{Ca}^{2+}$ sensor STIM and the plasma membrane $\mathrm{Ca}^{2+}$ entry channel Orai as the core components. Notably, live-cell imaging of STIM upon ER $\mathrm{Ca}^{2+}$ store depletion showed marked clustering of STIM in the ER and that it physically interacted with Orai at contact sites [35]. Work in the timeframe of this review has furthered the view that SOCE is subject to complex regulation. Septins emerged as key coordinators of SOCE [36]. Septins are highly regulated filament forming proteins laid down on membranes and in cytoplasmic meshes to create intracellular boundaries that can act as diffusion barriers. An siRNA screen for regulators of NFAT translocation to the nucleus (which is dependent of SOCE), identified septins 3, 4 and 5. Depletion of septins inhibits SOCE by preventing Orai clustering. Both septins and $\mathrm{PI}(4,5) \mathrm{P}_{2}$, which septins bind, redistribute during store depletion, with septin forming a boundary around a region rich in $\mathrm{PI}(4,5) \mathrm{P}_{2}$. To turn off SOCE, it has now been shown that Orai-STIM1 clusters move into this physically demarcated signaling microdomain, and there $\mathrm{Ca}^{2+}$ entry through Orai is inhibited by recruitment of SARAF, an integral ER protein [37]. Yet more organizers of SOCE micro-domains such as STIMATE/TMEM110 [38•,39•] are emerging.

Also there is new insight into the function of extended-synaptotagmins (E-Syts), ER-plasma membrane contact site proteins that respond to $\mathrm{Ca}^{2+}$ entry. E-Syt1-3 (yeast homologs called tricalbins) are integral ER proteins that bridge across contacts to the plasma membrane, where they bind anionic lipids and $\mathrm{Ca}^{2+}$ with multiple $\mathrm{C} 2$ domains $[30,40,41 \bullet]$. The expression level of E-Syts correlates with contact site size, but E-Syts also contain a lipid transfer domain that may exchange lipids between the ER and plasma membrane, so they are not merely tethers. E-Syt1 has two unique functions not shared by E-Syt2 and E-Syt3 that have now been studied in detail. The first is that the third of the five C2 domains in E-Syt1 binds the plasma membrane only when cytosolic $\mathrm{Ca}^{2+}$ levels rise, such as happens during induction of SOCE; this may narrow the contact site gap $[14 \bullet, 42]$. The second is that tethering of micro-domains rich in both $\mathrm{PI}(4,5) \mathrm{P}_{2}$ and Orai-STIM1 requires only E-Syt1, not E-Syt2 or E-Syt3 [37].

Summating all these findings, it appears that there is lateral compartmentalisation of different stages of the SOCE pathway within contact sites, with $\mathrm{Ca}^{2+}$ entry through $\mathrm{PI}(4,5) \mathrm{P}_{2}$-poor micro-domains regulating dynamics of $\mathrm{PI}(4,5) \mathrm{P}_{2}$-rich micro-domains in close proximity (Figure 2$)$. The functional consequence of such regulation remains to be established.

\section{Cyclic AMP at contacts}

The classical second messenger cyclic AMP (cAMP) has been proposed to signal at precise intracellular locations [43], but as yet membrane contact sites have not been implicated. cAMP is synthesized by ten adenylate cyclases, some soluble, others membrane bound, in response to G-protein coupled receptor activation. Compartmentalization is achieved by A-kinase anchoring proteins (AKAPs) which scaffold large complexes that include combinations of cAMP-dependent kinases (PKA), their targets, and both protein phosphatases and phosphodiesterases to terminate signaling [44]. The relationship of cAMP signaling with membrane contacts is that two AKAPs have been shown to bind to VAP $[45,46 \bullet]$, which is a common target on the ER for membrane contact site proteins [2]. AKAP220 (alternative name AKAP11) mediates crosstalk between PKA and GSK3 $\beta$ [47]. AKAP110 (alternative names AKAP3, SKIP, SPKAP) is in various locations including the mitochondrial matrix [48], where it may mediate the effects of intra-mitochondrial cyclic AMP on ATP production [49]. The interactions of these AKAPs with VAP positions cAMP signaling at membrane contact sites with the ER, opening up a new aspect of second messenger signaling at contact sites.

\section{Outlook}

Membrane contact sites are the only places where molecular machines on two different compartmental membranes can directly interact. They are possibly involved in any signaling event 
that involves multiple compartments. The involvement of multiple compartments has turned out to be important in many unexpected places (for example, [50]). Here we define contact sites as having two constituent membranes (Box 1), and we have focussed on contacts of either plasma membrane or acidic organelles (lysosome or degradative vacuole) with the ER. However, a new theme is that contacts may be more than two-way. A good example is the role of mitochondria, which form contacts not only with the ER [3-5], possibly more than one type [51], but also directly with both the plasma membrane [52] and with acidic organelles [53]. This indicates that multiple contacts on three or more compartments may interact with one another.

\section{Acknowledgements:}

We would like to thank Tony Lai for advice and Maya Schuldiner for inspiration. TPL's lab is supported by the BBSRC, the MRC and by the Marie-Curie ITN 'Sphingonet' (FP7, Grant no. 289278). Work in SP's lab is funded by the BBSRC and Parkinson's UK. The authors declare that there are no conflicts of interest. We apologise to all those whose work on this subject could not be cited because of lack of space. 


\section{BOX 1: Definitions}

\section{Membrane Contact Sites}

One definition for membrane contact sites might include only those with an intermembrane gap within a defined range, assumed to be $10-30 \mathrm{~nm}$, and if specific proteins and functions are known. However, we suggest a much wider definition of contacts as places where organelles are found so close together (without ever fusing) that proteins in each membrane form complexes that bridge the gap to create a functionally significant relationship. The inter-membrane gap is not constrained by the 10-30 nm norm. In the particular, the upper limit for gaps is not yet known. Some bona fide membrane contact site proteins can reach $\geq 100 \mathrm{~nm}$, for example Ist2p and STIM proteins [54], so this is the current upper limit of contact site width, but it could yet be outdone. Also, the combinations of organelles that might form contact sites is far wider than first thought, and recent work suggests that they may be found wherever two organelles share biochemical functions [50].

\section{Lipid transfer proteins}

Lipid transfer proteins have the in vitro ability to solubilise lipids and can move them from donor to acceptor liposomes [55]. Typically they have the form of a box with a mobile lid, both with a largely hydrophobic lining [2]. The lipids that are solubilised in vivo appear to be dominated by one or two species, but close examination reveals highly complex mixtures [56•], the significance of which is not yet known. It is considered likely that lipid binding will always affect the outside conformation of the domain, even with the lid re-closed, so it is accepted that all lipid transfer domains can generate signals by acting as lipid sensors. Some lipid transfer proteins have such strong effects on signaling pathways away from contact sites that lipid sensing is proposed to be more significant than lipid transfer [57]. This has led to a more revolutionary idea that presumed lipid traffic in vivo does not occur according to current hypotheses describing lipid transfer proteins, all of which are based on over-literal interpretations of the in vitro activity [55]. The solution to this controversy lies in testing how lipids are actually handled inside cells $[18 \bullet \bullet, 19 \bullet \bullet]$, and if lipid transfer proteins interact with proposed downstream components in signaling pathways, such as PI kinases.

\section{LEGENDS}

Figure 1. Proposed actions of RdgB/Nir proteins to resupply lipid during PIP hydrolysis

Signaling is initiated by $\mathrm{PI}(4,5) \mathrm{P}_{2}$ hydrolysis by PLC to diacylglycerol (DAG) in the plasma membrane (black arrow), with release of $\mathrm{IP}_{3}$. Intense signaling can only be maintained by resynthesis of $\mathrm{PI}(4,5) \mathrm{P}_{2}$ by the pathway DAG $\rightarrow$ phosphatidic acid (PA) $\rightarrow$ CDP-DAG $\rightarrow$ phosphatidylinositol (PI) $\rightarrow$ PI 4-phosphate $\left({ }^{4} \mathrm{P}\right) \rightarrow \mathrm{PI}(4,5) \mathrm{P}_{2}\left({ }^{45} \mathrm{P}_{2}\right)$. Among the enzymes (yellow arrows) for each of the five steps, three are on the plasma membrane: DAG kinase, PI 4-kinase and PIP 5-kinase. Two enzymes are in the ER: CDP-DAG synthase and PI synthase. The lipid solubilizing domains of RdgB and Nir2 transfer both PA from plasma membrane to ER, and PI in the opposite direction (blue and purple arrows respectively). The domains also can transfer PC (not shown), but how this integrates with PI/PA transfer is not yet known. RdgB/Nir2 also targeting both membranes: (i) a FFAT motif " $\mathrm{f}$ " that binds VAP "V", which is an integral ER protein; (ii) its C-terminal domains bind PA [20] and a second plasma membrane determinant $(*)$ that has been identified as DAG [19••]. Since both PA and DAG accumulate on the plasma membrane soon after PLC is activated, proteins in this family are recruited specifically when they are needed [21•]. Because PA is a building block contained within all other glycerophospholipids, including PI and PC, it is likely that PA can fit into all PI/PC transfer proteins. However, only a minority of such proteins efficiently transfer PA, possibly because they specifically interact with the shared 1phosphate. A proposed variation in this cycle is that RdgB/Nir2 and related PI transfer proteins present resynthesised PI directly to PI 4-kinase (dotted blue arrow).

Figure 2. Regulation of store-operated $\mathrm{Ca}^{2+}$ entry (SOCE) at ER-plasma membrane contact sites

Depletion of ER stores results in interactions between STIM1 in the ER and Orai1 in the plasma membrane (PM) resulting in substantial $\mathrm{Ca}^{2+}$ influx (left, indicated by yellow fill). This is regulated by translocation (large dotted black arrow) of STIM1/Orai1 from a PI(4,5) $\mathrm{P}_{2}$-poor region into a PI(4,5) $\mathrm{P}_{2}$ rich region of the PM demarcated by Septins (right, level of PI $(4,5) \mathrm{P}_{2}$ indicated by red fill). Here, STIM1 
interacts with SARAF which in turn promotes $\mathrm{Ca}^{2+-d e p e n d e n t ~ i n a c t i v a t i o n ~ o f ~ O r a i 1 ~ t h e r e b y ~ t e m p e r i n g ~}$ $\mathrm{Ca}^{2+}$ influx. This plasma membrane microdomain is stabilized by E-Syt1, which targets here by coincidence detection of $\mathrm{Ca}^{2+}$ and $\mathrm{PI}(4,5) \mathrm{P}_{2}$ that are bound by its third and fifth $\mathrm{C} 2$ domains (indicated by yellow and red edges respectively).

\section{References and recommended reading}

Papers of particular interest published within the period of review have been highlighted as:

- of special interest

•• of outstanding interest

[1] Burgoyne T, Patel S, Eden ER: Calcium signaling at ER membrane contact sites. Biochim Biophys Acta 2015, 1853:2012-2017.

[2] Prinz WA: Bridging the gap: membrane contact sites in signaling, metabolism, and organelle dynamics. J Cell Biol 2014, 205:759-769.

[3] Bernhard W, Rouiller C: Close topographical relationship between mitochondria and ergastoplasm of liver cells in a definite phase of cellular activity. J Biophys Biochem Cytol 1956, 2:73-78

[4] Block BA, Imagawa T, Campbell KP, Franzini-Armstrong C: Structural evidence for direct interaction between the molecular components of the transverse tubule/sarcoplasmic reticulum junction in skeletal muscle. J Cell Biol 1988, 107:2587-2600.

[5] Wirtz KW, Zilversmit DB: Exchange of phospholipids between liver mitochondria and microsomes in vitro. J Biol Chem 1968, 243:3596-3602.

[6] Manford AG, Stefan CJ, Yuan HL, Macgurn JA, Emr SD: ER-to-plasma membrane tethering proteins regulate cell signaling and ER morphology. Dev Cell 2012, 23:1129-1140.

[7] Pan X, Roberts P, Chen Y, Kvam E, Shulga N, Huang K, Lemmon S, Goldfarb DS: Nucleus-vacuole junctions in Saccharomyces cerevisiae are formed through the direct interaction of Vac8p with Nvj1p. Mol Biol Cell 2000, 11:2445-2457.

[8] Berridge MJ, Bootman MD, Roderick HL: Calcium signalling: dynamics, homeostasis and remodelling. Nat Rev Mol Cell Biol 2003, 4:517-529.

[9] Rizzuto R, De Stefani D, Raffaello A, Mammucari C: Mitochondria as sensors and regulators of calcium signalling. Nat Rev Mol Cell Biol 2012, 13:566-578.

[10] Whatmore J, Wiedemann C, Somerharju P, Swigart P, Cockcroft S: Resynthesis of phosphatidylinositol in permeabilized neutrophils following phospholipase Cbeta activation: transport of the intermediate, phosphatidic acid, from the plasma membrane to the endoplasmic reticulum for phosphatidylinositol resynthesis is not dependent on soluble lipid carriers or vesicular transport. Biochem J 1999, 341 ( Pt 2):435-444.

[11] Cunningham E, Thomas GM, Ball A, Hiles I, Cockcroft S: Phosphatidylinositol transfer protein dictates the rate of inositol trisphosphate production by promoting the synthesis of PIP2. Curr Biol 1995, 5:775-783.

[12] Bochud A, Conzelmann A: The active site of yeast phosphatidylinositol synthase Pis1 is facing the cytosol. Biochim Biophys Acta 2015, 1851:629-640.

[13] Tamura Y, Harada Y, Nishikawa S, Yamano K, Kamiya M, Shiota T, Kuroda T, Kuge O, Sesaki H, Imai $\mathrm{K}$, et al.: Tam41 is a CDP-diacylglycerol synthase required for cardiolipin biosynthesis in mitochondria. Cell Metab 2013, 17:709-718.

-[14] Fernandez-Busnadiego R, Saheki Y, De Camilli P: Three-dimensional architecture of extended synaptotagmin-mediated endoplasmic reticulum-plasma membrane contact sites. Proc Natl Acad Sci U S A 2015, 112:E2004.

Electron microscopic tomography of vitrified specimens shows that membrane contact sites in neurons are of two different types, one corresponding to contacts seen in tissue culture cells driven 
to have high levels of contacts containing STIM1 and Orai1, and another corresponding to contacts in cells over-expressing E-Syts. Each type of contact has a characteristic width and a characteristic appearance of proteins within the 15-27 nm contact site gap, and there were changes upon cell stimulation that correspond to activation of an extra C2 domain within E-Syt1, leading to binding at a closer range.

[15] Vihtelic TS, Goebl M, Milligan S, O'Tousa JE, Hyde DR: Localization of Drosophila retinal degeneration B, a membrane-associated phosphatidylinositol transfer protein. J Cell Biol 1993, 122:1013-1022.

[16] Chang CL, Hsieh TS, Yang TT, Rothberg KG, Azizoglu DB, Volk E, Liao JC, Liou J: Feedback regulation of receptor-induced Ca2+ signaling mediated by E-Syt1 and Nir2 at endoplasmic reticulum-plasma membrane junctions. Cell Rep 2013, 5:813-825.

[17] Garner K, Hunt AN, Koster G, Somerharju P, Groves E, Li M, Raghu P, Holic R, Cockcroft S: Phosphatidylinositol transfer protein, cytoplasmic 1 (PITPNC1) binds and transfers phosphatidic acid. J Biol Chem 2012, 287:32263-32276.

-•[18] Yadav S, Garner K, Georgiev P, Li M, Gomez-Espinosa E, Panda A, Mathre S, Okkenhaug H, Cockcroft S, Raghu P: RDGBalpha, a PI-PA transfer protein regulates G-protein coupled PtdIns(4,5)P2 signalling during Drosophila phototransduction. J Cell Sci 2015, epub:jcs.173476.

Yadav et al. (2015) and Kim et al. (2015) [19] both came to similar conclusions that RdgB in flies and its human homolog Nir2 respectively carry out PI/PA exhcange at plasma membrane-ER contacts. Yadav et al. showed that the RdgB domain also transfers PA in vitro. Kim et al. demonstrated that Nir2 is required to avoid precursor build up for both lipid transfer steps, and also that the Cterminal domains mediate targeting to plasma membrane-ER contacts. These papers together with the known locations of the enzymes required for PIP consumption and resynthesis establish an absolute requirement for this lipid transfer protein to transfer lipids in vivo. This provides part of the answer to a question raised elsewhere [55]: these PI transfer proteins (PITPs) do move lipids from one place to another. Also see Chang et al. [21] for a nuanced study of Nir3.

••[19] Kim YJ, Guzman-Hernandez ML, Wisniewski E, Balla T: Phosphatidylinositol-Phosphatidic Acid Exchange by Nir2 at ER-PM Contact Sites Maintains Phosphoinositide Signaling Competence. Dev Cell 2015, 33:549-561.

See note for Yadav et al. (2015) [18].

[20] Kim S, Kedan A, Marom M, Gavert N, Keinan O, Selitrennik M, Laufman O, Lev S: The phosphatidylinositol-transfer protein Nir2 binds phosphatidic acid and positively regulates phosphoinositide signalling. EMBO Rep 2013, 14:891-899.

-[21] Chang CL, Liou J: Phosphatidylinositol 4,5-Bisphosphate Homeostasis Regulated by Nir2 and Nir3 Proteins at Endoplasmic Reticulum-Plasma Membrane Junctions. J Biol Chem 2015, 290:14289-14301.

This paper can be seen as a companion to Yadav et al. (2015) [18] and Kim et al. (2015) [19]. Nir3, a paralog of Nir2 found only in vertebrates, was found to work alongside it, but to have different sensitivities: translocation to contacts by Nir3 is much more sensitive because its C-terminal domains have a higher affinity for PA; also its PI transfer domain is lable to transfer PA, but is slower than Nir2. These modulations allow Nir3 to function after low level PIP hydrolysis, while Nir2 is adapted to faciliate maximal stimulation.

[22] Schaaf G, Ortlund EA, Tyeryar KR, Mousley CJ, Ile KE, Garrett TA, Ren J, Woolls MJ, Raetz CR, Redinbo MR, et al.: Functional anatomy of phospholipid binding and regulation of phosphoinositide homeostasis by proteins of the sec14 superfamily. Mol Cell 2008, 29:191206.

[23] Patel S, Churchill GC, Galione A: Coordination of Ca2+ signalling by NAADP. Trends Biochem Sci 2001, 26:482-489.

[24] Patel S: Function and dysfunction of two-pore channels. Sci Signal 2015, 8:re7.

[25] Kilpatrick BS, Eden ER, Schapira AH, Futter CE, Patel S: Direct mobilisation of lysosomal Ca2+ triggers complex Ca2+ signals. J Cell Sci 2013, 126:60-66. 
[26] Brailoiu E, Rahman T, Churamani D, Prole DL, Brailoiu GC, Hooper R, Taylor CW, Patel S: An NAADP-gated two-pore channel targeted to the plasma membrane uncouples triggering from amplifying Ca2+ signals. J Biol Chem 2010, 285:38511-38516.

[27] Patel S, Brailoiu E: Triggering of Ca2+ signals by NAADP-gated two-pore channels: a role for membrane contact sites? Biochem Soc Trans 2012, 40:153-157.

[28] Fameli N, Ogunbayo OA, van Breemen C, Evans AM: Cytoplasmic nanojunctions between lysosomes and sarcoplasmic reticulum are required for specific calcium signaling. F1000Res 2014, 3:93.

[29] Capel RA, Bolton EL, Lin WK, Aston D, Wang Y, Liu W, Wang X, Burton RB, Bloor-Young D, Shade KT, et al.: Two pore channels (TPC2s) and nicotinic acid adenine dinucleotide phosphate (NAADP) at lysosomal-sarcoplasmic reticular junctions contribute to acute and chronic betaadrenoceptor signaling in the heart. J Biol Chem 2015, 10.1074/jbc.M115.684076.

[30] Toulmay A, Prinz WA: A conserved membrane-binding domain targets proteins to organelle contact sites. J Cell Sci 2012, 125:49-58.

[31] Wong LH, Levine TP: Lipid transfer proteins do their thing anchored at membrane contact sites... but what is their thing? Biochem Soc Trans 2016, in press.

••[32] Henne WM, Zhu L, Balogi Z, Stefan C, Pleiss JA, Emr SD: Mdm1/Snx13 is a novel ERendolysosomal interorganelle tethering protein. J Cell Biol 2015, 210:541-551.

A genome-wide screen for new genes involved in traffic to the yeast vacuole was used to idnetify Mdm1p, the yeast homolog of human Snx13/14/15, and its previously unstudied homolog Ydr179w-ap (Nvj3p) as novel components of the nucleus-vacuole junction. One domain is common to all these proteins: the PXA domain, which has no known function. Mdm1 and human

Snx13/14/15 have extra domains: transmembrane helices that are embedded in the outer nuclear envelope, and PX domains that target the vacuole by binding PI(3)P. Loss of Mdm1p function (as per Snx14 mutations that cause complex and severe human neurological problems) cause defects in sphinoglipid metabolism, which is one of the proposel functions of the NVJ. Both of the newly studied yeast components are seen at contacts between cytoplasmic ER tubules and the vacuole, in particular when Nvj1p is deleted. This second class of contacts between elements of the ER and the degradative vacuole has never been described formally before, and the name Vacuolar non-NVJ cytoplasmic ER (VancE) contacts has been proposed [40].

[33] Elbaz-Alon Y, Eisenberg-Bord M, Shinder V, Stiller SB, Shimoni E, Wiedemann N, Geiger T, Schuldiner M: Lam6 Regulates the Extent of Contacts between Organelles. Cell Rep 2015, 12:714.

[34] Thomas AC, Williams H, Seto-Salvia N, Bacchelli C, Jenkins D, O'Sullivan M, Mengrelis K, Ishida M, Ocaka L, Chanudet E, et al.: Mutations in SNX14 cause a distinctive autosomal-recessive cerebellar ataxia and intellectual disability syndrome. Am J Hum Genet 2014, 95:611-621.

[35] Liou J, Kim ML, Heo WD, Jones JT, Myers JW, Ferrell JE, Jr., Meyer T: STIM is a Ca2+ sensor essential for Ca2+-store-depletion-triggered Ca2+ influx. Curr Biol 2005, 15:1235-1241.

[36] Sharma S, Quintana A, Findlay GM, Mettlen M, Baust B, Jain M, Nilsson R, Rao A, Hogan PG: An siRNA screen for NFAT activation identifies septins as coordinators of store-operated Ca2+ entry. Nature 2013, 499:238-242.

[37] Cao X, Choi S, Maleth JJ, Park S, Ahuja M, Muallem S: The ER/PM microdomain, PI(4,5)P(2) and the regulation of STIM1-Orai1 channel function. Cell Calcium 2015, 58:342-348.

-[38] Jing J, He L, Sun A, Quintana A, Ding Y, Ma G, Tan P, Liang X, Zheng X, Chen L, et al.: Proteomic mapping of ER-PM junctions identifies STIMATE as a regulator of $\mathrm{Ca}(2)(+)$ influx. Nat Cell Biol 2015, 17:1339-1347.

This paper uses an elegant spatially restricted proteomic approach to identify a physical interaction between TMEM110 (renamed STIMATE) and STIM1 which is mapped to the coiled-coil region of STIM1. This region is known to mediate a conformational switch that releases the SOAR/CAD domain of STIM1 to allow gating of Orai. STIMATE may therefore promote Ca2+ entry by stabilizing short term transition states upon store depletion as well long term stabilization of ER-PM junctions - see [52]. 
-[39] Quintana A, Rajanikanth V, Farber-Katz S, Gudlur A, Zhang C, Jing J, Zhou Y, Rao A, Hogan PG: TMEM110 regulates the maintenance and remodeling of mammalian ER-plasma membrane junctions competent for STIM-ORAI signaling. Proc Natl Acad Sci U S A 2015, 112:E7083-7092.

This paper and [51] provide insight into the mechanism of action of TMEM110, a protein previously identified in a siRNA screen for regulators of NFAT translocation. TMEM110 is identified as a positive regulator of SOCE. Knockdown/knockout studies reveal a substantial reduction in cortical ER, ER-PM junctions and the formation of STIM1 clusters upon store depletion.

[40] Giordano F, Saheki Y, Idevall-Hagren O, Colombo SF, Pirruccello M, Milosevic I, Gracheva EO, Bagriantsev SN, Borgese N, De Camilli P: PI(4,5)P(2)-dependent and Ca(2+)-regulated ER-PM interactions mediated by the extended synaptotagmins. Cell 2013, 153:1494-1509.

-[41] Schauder CM, Wu X, Saheki Y, Narayanaswamy P, Torta F, Wenk MR, De Camilli P, Reinisch KM: Structure of a lipid-bound extended synaptotagmin indicates a role in lipid transfer. Nature 2014, 510:552-555.

A crystal structure of E-Syt2 showed that it contains the tube-forming head-to-head dimeric lipid transfer domains predicted by Kopec et al. (Bioinformatics, 2010, vol 26, pp1927-31), but with two important additional discoveries: (1) Four phospholipids are bound to each protein dimer, and lipids extracted from mammalian cells are representative of the inner leaflet of the plasma membrane, confirming a prediction that E-Syts bind only acyl chains, with headgroups projecting out of the protein so that they are not subject to any selection. (2) The overall length of the lipid carrying tube is $<10 \mathrm{~nm}$ and its ends are hydrophilic; this severly undermines a model where E-syts and related proteins at contacts form tubular bridges for lipids to flow through across contact sites, and an alternative model is suggested where lipids are shuttled across contacts four at a time. It is intriguing to question how any phospholipid gradient between ER and plasma membrane is maintained if E-Syts can exchange them non-specifically.

[42] Idevall-Hagren O, Lu A, Xie B, De Camilli P: Triggered Ca2+ influx is required for extended synaptotagmin 1-induced ER-plasma membrane tethering. Embo J 2015, 34:2291-2305.

[43] Lefkimmiatis K, Zaccolo M: cAMP signaling in subcellular compartments. Pharmacol Ther 2014, 143:295-304.

[44] Wong W, Scott JD: AKAP signalling complexes: focal points in space and time. Nat Rev Mol Cell Biol 2004, 5:959-970.

[45] Mikitova V, Levine TP: Analysis of the key elements of FFAT-like motifs identifies new proteins that potentially bind VAP on the ER, including two AKAPs and FAPP2. PLoS One 2012, 7:e30455.

-[46] Huttlin EL, Ting L, Bruckner RJ, Gebreab F, Gygi MP, Szpyt J, Tam S, Zarraga G, Colby G, Baltier K, et al.: The BioPlex Network: A Systematic Exploration of the Human Interactome. Cell 2015, 162:425-440.

A high-throughput interomics project was reported oringinally with 26,000 interactions, and then later expanded with an on-line update that includes $\geq 56,000$ interactions from $>5000$ tagged proteins. The quality of the data was sufficiently high to identify likely functions and locations for proteins of unknown function. A detailed study was carried out for the interactions of VAP (standing for VAMP-associated protein). This is a key membrane contact site protein as it is the ER receptor for many proteins that target contact sites, and is important for many ER functions, as well as being implicated in motor neurone disease (also called amyotrophic lateral sclerosis, ALS) [57]. AKAP110 was among the interactors, as previously predicted from its conserved FFAT motif [55], along with several PKA regulatory subunits that likely bind to it. This places cAMP signalling at contact sites.

[47] Whiting JL, Nygren PJ, Tunquist BJ, Langeberg LK, Seternes OM, Scott JD: Protein Kinase A Opposes the Phosphorylation-dependent Recruitment of Glycogen Synthase Kinase 3beta to A-kinase Anchoring Protein 220. J Biol Chem 2015, 290:19445-19457.

[48] Means CK, Lygren B, Langeberg LK, Jain A, Dixon RE, Vega AL, Gold MG, Petrosyan S, Taylor SS, Murphy AN, et al.: An entirely specific type I A-kinase anchoring protein that can sequester two molecules of protein kinase A at mitochondria. Proc Natl Acad Sci U S A 2011, 108:E12271235. 
[49] Di Benedetto G, Scalzotto E, Mongillo M, Pozzan T: Mitochondrial Ca(2)(+) uptake induces cyclic AMP generation in the matrix and modulates organelle ATP levels. Cell Metab 2013, 17:965-975.

[50] Chu BB, Liao YC, Qi W, Xie C, Du X, Wang J, Yang H, Miao HH, Li BL, Song BL: Cholesterol transport through lysosome-peroxisome membrane contacts. Cell 2015, 161:291-306.

[51] Lahiri S, Chao JT, Tavassoli S, Wong AK, Choudhary V, Young BP, Loewen CJ, Prinz WA: A conserved endoplasmic reticulum membrane protein complex (EMC) facilitates phospholipid transfer from the ER to mitochondria. PLoS Biol 2014, 12:e1001969.

[52] Lackner LL, Ping H, Graef M, Murley A, Nunnari J: Endoplasmic reticulum-associated mitochondria-cortex tether functions in the distribution and inheritance of mitochondria. Proc Natl Acad Sci U S A 2013, 110:E458-467.

[53] Ungermann C: vCLAMPs - an intimate link between vacuoles and mitochondria. Current Opinion in Cell Biology 2015, 35:30-36.

[54] Kralt A, Carretta M, Mari M, Reggiori F, Steen A, Poolman B, Veenhoff LM: Intrinsically disordered linker and plasma membrane-binding motif sort Ist2 and Ssy1 to junctions. Traffic 2015, 16:135-147.

[55] Grabon A, Khan D, Bankaitis VA: Phosphatidylinositol transfer proteins and instructive regulation of lipid kinase biology. Biochim Biophys Acta 2015, 1851:724-735.

-[56] Schrick K, Bruno M, Khosla A, Cox PN, Marlatt SA, Roque RA, Nguyen HC, He C, Snyder MP, Singh D, et al.: Shared functions of plant and mammalian StAR-related lipid transfer (START) domains in modulating transcription factor activity. BMC Biol 2014, 12:70.

Heterologous lipid transfer domains of the StART family were expressed in yeast as fusions placed in between a GAL4 DNA binding domain from yeast and the activation domain of herpes VP16. The resulting constructs were transcriptional stimulators in a StART-dependent fashion. Although the details of how StART domains activate this construct are not clear, the natural ligands for a number of active constructs were studied by mass spectrometry of protein recovered from cells.

Interestingly, a wide range of lipidic ligands (5-40) was found in all cases, with hierarchical clustering required to identify the classes of ligands specific for each domain.

[57] Mousley CJ, Yuan P, Gaur NA, Trettin KD, Nile AH, Deminoff SJ, Dewar BJ, Wolpert M, Macdonald JM, Herman PK, et al.: A sterol-binding protein integrates endosomal lipid metabolism with TOR signaling and nitrogen sensing. Cell 2012, 148:702-715. 

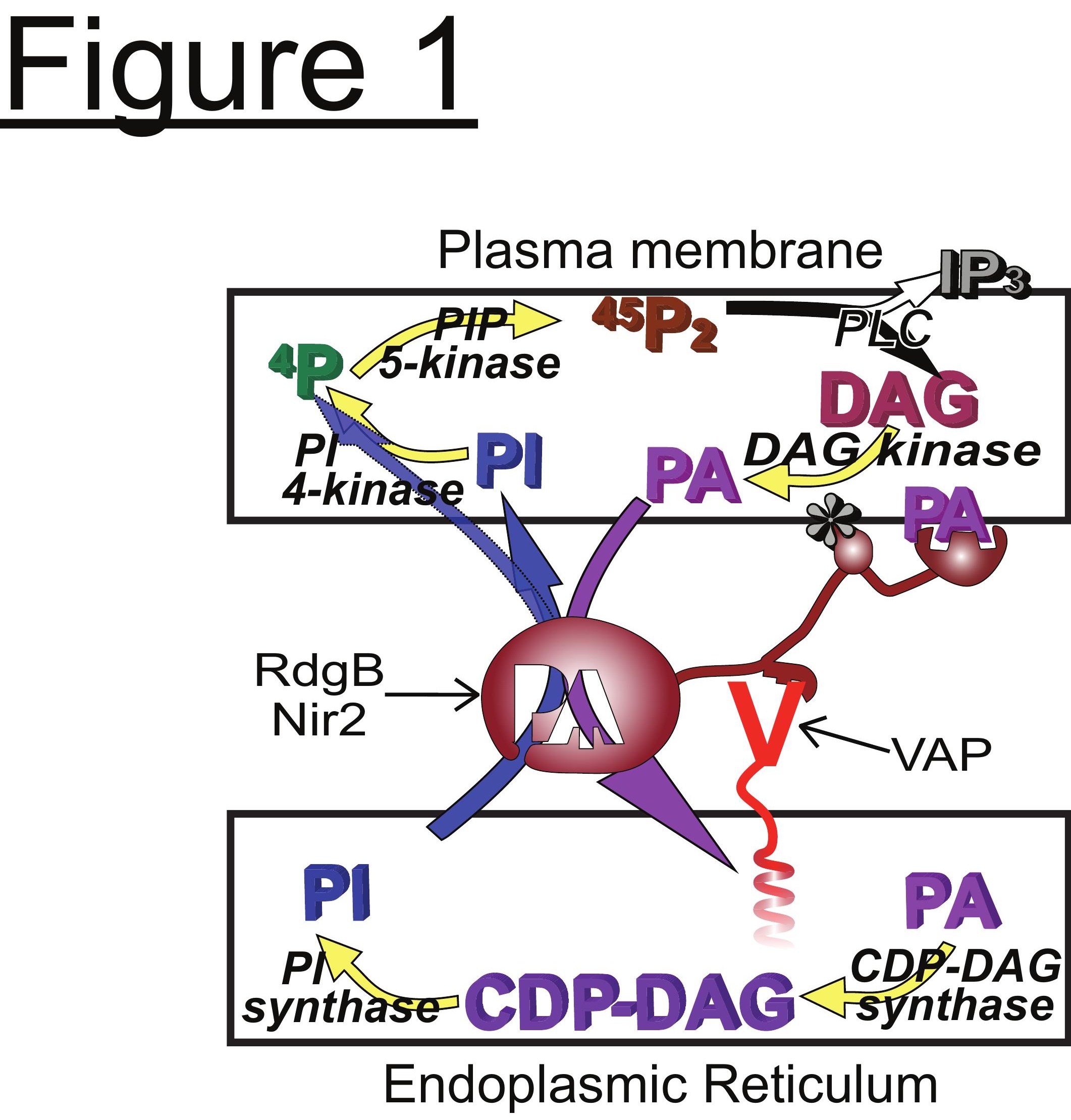


\section{Figure 2}

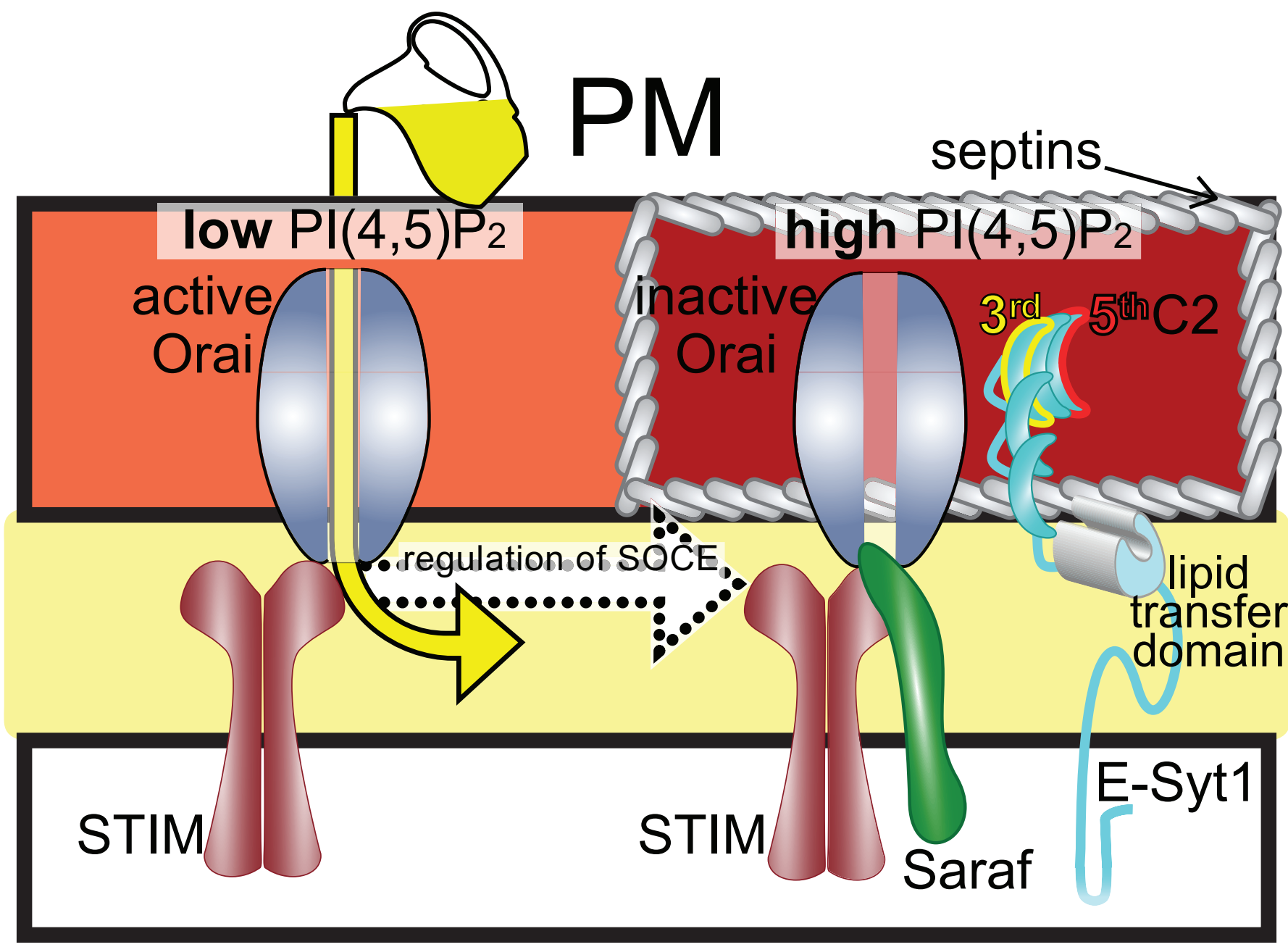

